\title{
Editorial Volume 4 (2)
}

\section{Introduction}

We are delighted to present the eighth edition of Exchanges: the Warwick Research Journal. This edition completes the fourth volume of the journal and continues to promote exciting interdisciplinary research and scholarship from researchers at all stages of their careers.

Every year Exchanges continues to grow in reputation as a peer-reviewed, open access, interdisciplinary journal. This is evidenced by the large number of high-quality submissions we continue to attract. This edition includes articles from a range of disciplines, including History of Science, Law, Theatre Studies, Food Studies, Politics and International Relations, Sociology, Economics, History of Art, Film Studies, Literary Studies, Philosophy, Engineering, and Mathematics. All of the articles published in this edition highlight the importance of breaking down traditional disciplinary boundaries. We hope that they will offer productive exchanges between different academic disciplines.

\section{Exchange, dialogue, and debate}

Exchanges strives to promote dialogue and debate through the articles and pieces it publishes. Both conversations in this edition focus on interdisciplinarity. 'Ideals and Practices of Rationality - An Interview with Lorraine Daston' is concerned with the history of science. As Professor Daston notes, historians of science often come from other disciplines, '[taking] an ex-ante trajectory through the sciences, sometimes through philosophy, sometimes through history, sometimes through sociology' (176). 'Acting Law / Law Acting: A Conversation with Dr Felix Nobis and Professor Gary Watt' considers the intersection between law and performance.

Michael Bycroft talks to Professor Lorraine Daston, recipient of the Sarton Medal of the History of Science in 2012, about 'historical epistemology'. To echo the theme of collaboration, Professor Daston shares her experience in collaborative research, especially at the Max Planck Institute for the History of Science in Berlin, where she is a director. In particular, she discusses her co-authored monographs, which 'grew out of conversations [...] that straddled the conventional periodisation within the history of science' (181), and edited collections, which resulted from collaborative research projects 'that none of us can 
do alone, that can reach across many, many centuries' (182). Aware of the demands on early career researchers to produce independent research, she suggests the way in which they might also participate in collaborative projects. Moreover, this conversation piece touches upon Professor Daston's current project, entitled The Sciences of the Archives: Big Science, Big Humanities, and the Pathos of Positivism. If you find the discussion here interesting and would like to know more about the project, you could listen to her Distinguished Lecture given on 6 March 2017 at the University of Warwick at http://www2.warwick.ac.uk/newsandevents/events/distinguishedlecture /lorrainedaston/.

To 'understand law's performance and the performance of law', Sean Mulcahy brings together 'an actor who has sometimes played lawyers [Dr Felix Nobis] and a law professor who has sometimes acted [Professor Gary Watt]' (190). The interview covers a wide range of topics, such as the 'complete myth of the scientific predictability of law' (191), truth, voice and gesture, dress, space and movement, and the connection with the audience. For example, the discussion about truth is illuminating. As Professor Watt points out, the judicial process is 'designed to perform a satisfactory outcome. [...] It is not a process of revealing truth, but a process of manufacturing proof' (193).

\section{Featured section: 'Collaboration'}

This edition offers a featured section on the theme of collaboration. Collaboration is essential to research within and across many disciplines. To tackle global issues and make a positive impact on society, researchers form networks not only with each other but also with a wide range of organisations at national and international levels to share expertise and resources (funding, specialised equipment, etc.) and to generate and analyse large amount of data. Despite the many opportunities it affords, collaborative work can sometimes be problematic. Exchanges reflects on the promises, achievements, and pitfalls of collaboration and how some of the shortcomings and issues arising from it can be overcome.

In 'Training Future Actors in the Food System', Kelly Reed et al. review the new collaborative cross-institutional, interdisciplinary training programme Innovative Food Systems Teaching and Learning (IFSTAL). Given that '[a] food system operates within, and is influenced by, social, political, economic and environmental contexts', they call for 'a more holistic way of thinking and working [which] is needed to provide an understanding of how problems are connected, their root causes, and 
where critical leverage points might be' $(202,203)$. The postgraduate programme is therefore designed to "create a generation of "food systems thinkers"' (203). Moreover, IFSTAL is an exemplar case study of innovative teaching, which is brought to the fore by the Teaching Excellence Fellowship (TEF).

Lucy Hatton reflects on the European Citizens' Initiative (ECI) Day 2016: Forging Change conference. Promoting participatory democracy, the $\mathrm{ECl}$ 'connects EU citizens with EU law-making institutions by enabling one million citizens to come together and invite the European Commission to propose a legal act' (219). With hindsight, the discussion of participatory democracy in 2016 was topical not only within the EU but also in a global context. In 2017, a year of elections in major European countries such as France, the UK, and Germany, and of possible referenda, the topic seems all the more pertinent. Interestingly, changes are often met with resistance, and the reluctance of the European Commission even to participate in the debate about change reveals that collaboration is not always possible, despite the fact that it is most needed. Lucy also emphasises the role of education in improving the EU's participatory democracy.

Both Eri Tsukamoto and Alice Eden review the Artists and Academics Exhibition held at Fargo Village in support of Coventry's City of Culture 2021 bid. The exhibition brought the two communities into dialogue, inviting the artists to create works that are inspired by academic research. While Tsukamoto, a doctoral researcher in Sociology, who participated in the event, reflected on her collaboration with the artist Frieda van de Poll in the broader context of the Research Excellence Framework (REF) and research impact, Eden, with her background in History of Art, discusses the experiences of viewing in light of her research on artists who 'considered the role of art for the public' (233) and her personal viewing experience of this exhibition with a local artist, Holly Dawes.

\section{Communicating cutting-edge interdisciplinary research}

The two remaining articles, one review article, and three critical reflection pieces published in this edition cover a wide range of topics.

To continue the celebration of 400 years of Shakespeare's legacy, Nike Jung examines Orson Welles's film adaptation Othello (1951) in relation to issues surrounding race in the US. She argues that Welles's early film The Hearts of Age (1934) 'illuminate[s] the understanding of Welles's use of blackface and whiteness in his later, controversially interpreted Othello', and that 'Welles highlighted critical themes already present in 
Shakespeare's source text, translating them into cinematic means that critically intervene in contemporary politics' (246).

Filling in a gap in literature on the emerging markets, Le Thi Hong Minh, Huynh Thi Cam Ha, and Dinh Thi Thu Hong study the exchange rate exposure of Southeast Asian stock markets, assessing the effects of exchange rate fluctuations on stock returns of security markets in Indonesia, Malaysia, Philippines, Singapore, Thailand, and Vietnam from 2009 to 2014. They have found the 'presence of asymmetric exchange rate exposure' (277). In the context of globalisation, they emphasise the importance of regional collaboration, such as the Asian Economic Community (AEC), which enhances resilience of local businesses.

In his review article, Josh Patel considers the 'biological turn' in history writing, which takes advantage of other disciplines such as human biology, Darwinism, neurobiology, and evolutionary-psychology. Acknowledging the new perspectives this interdisciplinarity brings, Patel, however, warns us that 'the new knowledge produced by the biological turn is construed as simply objective, and not politically, socially, and economically useful rhetoric' (292). For more meaningful interdisciplinary research, he, therefore, proposes 'even "deeper", multilateral collaboration' (293), and argues that History of Science, which views 'science as culture' (281), may offer an alternative. Interestingly, this 'biological turn' seems prevalent not only in History but also in the Humanities more generally.

In 'The Art of Supporting Decision-Making', Marco Cinelli shares with us his interviews with leading experts at the biennial conference of the International Society on Multiple Criteria Decision Making. Multiple Criteria Decision Making, Analysis and Aiding (MCDM/A) is 'a discipline that helps DMs making better decisions through mathematical modelling' (300). Cinelli discusses the strengths and challenges of MCDM/A, its applications (for example in Finance, Healthcare, Infrastructure, Logistics, Manufacturing, Marketing, Systems, and Sustainability) and integration into these areas.

Birgit Breidenbach's 'Grasping the Ineffable: Interdisciplinary Perspectives on Mood' offers a critical reflection on the Mood: Aesthetic, Psychological and Philosophical Perspectives conference held at Warwick in May 2016. Mood, as Breidenbach points out, is 'inherently interdisciplinary' (310); not surprisingly, the conference attracted researchers from a range of subjects such as philosophy, psychology, sociology, economics, literatures, and music. Key topics under discussion and debate include 'the spatiality and temporality of mood, the questions of its intentionality, responsibility and ethics, as well as its cultural specificity and cross-cultural capacity' (313-14). 
'A New World... Out of Nothing' is an interdisciplinary workshop that aims to convey the 'importance, elegance and beauty' (321) of (pure) mathematics to non-experts and to encourage dialogue with other disciplines. The organiser, Francesca lezzi, reflects on the event in the light of participants' written feedback. The workshop was a success because it attracted researchers from across the sciences and the humanities, raised awareness of mathematics in a wider context, and generated a rethink of the participants' own disciplines. It thus contributes greatly to bridging the divide between the 'two cultures', which brings us back to the interview with Professor Daston.

\section{Thanks}

Many thanks for your continued support of the journal through your readership and engagement with our articles. Readers play an important part in the life of the journal and we encourage you to share, comment on, enter into discussion, and ask questions about our articles and critical reflections. We hope you find this edition as stimulating and thoughtprovoking as we have, and hope that you enjoy reading pieces outside of your research specialisms.

Finally, we want to thank all of the peer reviewers who generously volunteered their time to read each of our submissions carefully and provided helpful, constructive feedback for our authors. We are truly grateful to Yvonne Budden, Head of Scholarly Communications at the University of Warwick, for her continued support and assistance with the development of the journal.

We look forward to the Fifth Anniversary edition, which will be published in October 2017. It will feature a conversation with Professor David Greenwood, who leads the Advanced Propulsion Systems at Warwick Manufacturing Group and the development of R\&D activities within the National Automotive Innovation Centre, and also a themed section on 'Movement'. 
The Editors

Dr Yuexi Liu (Senior Editor) / English

Dr James Amor / Engineering

Harbir Bal / Education

Dr Nicholas Collins / English

Dr Truong Quang Dinh / WMG

Dr Alice Eden / History of Art

Dr Stefania Ferrari / WMG

Dr Kelly Schmidtke / WBS

Daniel Silva / Politics and International Studies 